\title{
The Effect of Nitric Oxide with Minimal Stimulation on Patients with Polycystic Ovarian Syndrome
}

\author{
Kobre Amir Ajam ${ }^{1}$, Laya Farzadi ${ }^{*}$, Mohammad Nouri ${ }^{1}$, M M Sadagheani ${ }^{1}$
}

\section{Article History:}

Received 1 November 2013

Accepted 2 February 2014

Revised 17 January 2014

Available online 4 February 2014

Keywords:

Assisted Reproductive Techniques

Infertility

Nitric Oxide

\section{Corresponding Author:}

Laya Farzadi , Women's Reproductive Health Research Center, Tabriz Hospital, Tabriz University of Medical Sciences, Tabriz, Iran.

Tel: +989141153712

Email: Farzadl_29@yahoo.com

\begin{abstract}
Objectives: Infertility is one of the most prevalent problems among young couples especially in women with Polycystic Ovary Syndrome. In women, PCO is associated with lack of ovulation, abdominal obesity and insulin resistance. The aim of this study is to investigate the effect of nitric oxide with minimal stimulation in the treatment of infertility in women with PCOS.

Materials and Methods: In a randomized single blind clinical trial (RCT), 120 women who had polycystic ovarian syndrome (PCOS), were with PCOS who were eligible to be studied were divided into two groups; study group and a control group (60 patients in each group) and the effects of Nitric Oxide with minimal stimulation on the treatment of infertility were investigated in the patients who were being treated with IUI.

Results: The mean age of the patients, who were 21 to 35 years old, was $28.3 \pm 4.2$ years. In the study group, 43 cases $(71.7 \%)$ and in the control group, 52 cases $(86.7 \%)$ had ovulation. The difference between these two groups was statistically significant $(p=0.03)$. The successful pregnancy rate was 10 cases $(16.6 \%)$ in the study group and 8cases in the control group (13.3\%), however this difference was not statistically significant $(p=0.18)$. The difference between the two groups regarding mature follicles was not significant either $(p=0.27)$, but the difference regarding the endometrial thickness on the gonadotropin administration day was statistically significant $(\mathrm{p}=0.02)$.

Conclusion: According to the results and since there was not a significant difference between the two groups regarding the pregnancy rate, it can be concluded that employing nitric oxide tablets in addition to the common treatment, does not affect pregnancy rate in women with PCOS.
\end{abstract}

1 - Department of Gynecology- Alzahra Hospital- Faculty of Medicine, Tabriz University of Medical Sciences, Tabriz, Iran.

2- Women's Reproductive Health Research Center, Tabriz Hospital, Tabriz University of Medical Sciences, Tabriz, Iran. 


\section{Introduction:}

Polycystic Ovarian Syndrome (PCOS) is one of the most common endocrine disorders that affect about $5-10 \%$ of the women at the fertility age. The increase in the level of androgen is evident in $60-80 \%$ of the women with PCOS. Hyperandrogenism is an independent risk factor which can result in high blood pressure and an increased possibility of cardiovascular diseases in women with Polycystic Syndrome. Women with PCO and lack of ovulation, suffer from abdominal obesity and insulin resistance which leads to the increase in the possibility of the diabetes type 2 and cardiovascular diseases. Hyperandrogenism along with hirsutism and acne causes neuropsychiatric symptoms in these patients.

Polycystic Syndrome is the most common cause of oligomenorrhea and hirsutism in a way that $87 \%$ of the women with oligomenorrhea and $92 \%$ of the women with hirsutism show polycystic ovarian symptoms $(3,4)$. One of the other important disorders caused by polycystic ovarian syndrome is infertility. According to the previous research, the reason for the lack of ovulation in $83 \%$ of the infertile couples is polycystic ovarian syndrome and among $56 \%$ of the couples who had inexplicable fertility, $44 \%$ of them were reported to have polycystic ovary with ovulation periods (5). It seems that hyperandrogenism (acne, hirsutism, and alopecia) and menstrual disturbances (oligomenorrhea) are shown in 70.3 and $66.2 \%$ of the patients respectively. It is possible to diagnose the disease based on the sonography results, one or two clinical symptoms of the disease, or endocrine disorders in the form of increase in the serum concentration of $\mathrm{LH}$ and testosterone (6).Nitric oxide is a free radical gas whose effect does not last long and is made up of L- Arginine and is believed to be the main intervention in the most of the natural functions of the cardiovascular organs, reproductive organs, immune system and digestion (7). Based on the results of several studies, ovaries are able to synthesize nitric oxide and possibly this substance has a role in ovary steroid production, ovulation, and the destruction of the corpus Luteum (8). It seems that nitric oxide does not affect ovaries only when they are under natural conditions. Local effects of nitric oxide in polycystic ovary syndrome and with inexplicable reasons have been have been proved (9).There are studies that emphasize the effect of nitric oxide on steroid production in ovaries. During animal's sexual cycle, several changes in hormonal activities and in ovaries take place. Nitric oxide activity in the steroid production of the corpus Luteum is important (7). Under the cellular culture conditions, nitric oxide takes the aromatase enzyme activity under control and decreases estradiol production (10).

Since a lot of different studies have been carried out on this topic all over the world, and since it has never been investigated in the patients in our area, the present study investigated the effects of nitric oxide with minimal stimulation in the treatment of infertility in patients with polycystic ovarian syndrome.

\section{General purpose of the study:}

the general purpose of this study was to investigate the effect of nitric oxide with minimal stimulation in the treatment of infertility in patients with polycystic ovarian syndrome.

\section{Specific Purposes of the Study:}

The specific purposes of the study are:

1. Determine the effect of the nitric oxide with minimal stimulation on the number of the mature follicles on the day of the administration of HCG.

2. Determine the effect of the nitric oxide with minimal stimulation on the endometrial thickness on the day of the administration of HCG.

3. Determine the effect of the nitric oxide with minimal stimulation on the rate of ovulation by administering trans vaginal sonography on the day of IUI.

4. Determine the effect of the nitric oxide with minimal stimulation on the pregnancy rate.

\section{Practical Purpose of the Study:}

If this treatment is proved to be effective compared to the other common treatments, it can also be used to treat the patients with polycystic ovarian syndrome. 


\section{Study Hypotheses:}

1. Nitric oxide with minimal stimulation has no significant effects on the number of the mature follicles.

2. Nitric oxide with minimal stimulation has no significant effects on the endometrial thickness.

3. Nitric oxide with minimal stimulation has no significant effects on the rate of ovulation;

4. Nitric oxide with minimal stimulation has no significant effects on the pregnancy rate.

\section{Literature Review:}

Infertility and the assisted reproductive techniques in women suffering from PCOS: Infertility is defined as an inability to conceive after a year of regular sexual intercourse without contraception. Nowadays, $10-15 \%$ of the couples are infertile. Thus, it can be assumed that there are more than 80 million people in the world who are infertile. In Iran, it is estimated that of the population of 72 million people (18 million couples), more than 2 million people are faced with the infertility problems. World Health Organization (WHO) has declared infertility as an important problem in the reproductive health with many physical-mental and social dimensions (18). There are two main methods to treat infertility; intrauterine insemination of the sperm (IUI) along with controlled ovarian hyper stimulation $(\mathrm{COH})$, and assisted reproductive techniques (ART) (19).By the prescription of ovulation-stimulating drugs, ovaries are stimulated to grow follicles containing oocytes. Follicle maturation time is determined by the several sonographies of the ovaries and the measurement of the serum estradiol which start 8 days after the beginning of the treatment (20).

(22).There are studies which emphasize the role of nitric oxide in steroid production in the ovaries. Several changes happen in ovary and hormonal activities of animals in their sexual cycle. The role of nitric oxide role is important in steroid production of the corpus Luteum (7).Nitric oxide takes the enzyme system of the aromatase under control and reduces the production of estradiol (10).In a study entitled 'investigating the level of NO in the patients with and without PCOS' by Erdogan et al (2008) at the University of Ege in Izmir,
Turkey, the findings showed the level of NO did not differ significantly between the patients with PCOS and the control group (23).Findings of a similar study in Brazil by Nacul et al (2007) indicated that in patients with PCOS, the level of insulin has a significant negative correlation with the production of NO (24).In a research study in Turkey by Turkcuoglu et al (2010), the level of NO in the patients with PCOS was investigated. The results showed that the level of NO has a significant negative correlation with the level of fasting insulin and also the level on NO is related to the high level of fasting insulin in these patients (25).In some studies, it has been reported that NO is important to support the decidualization processes and has a protective role in implantation. In this study, it has been shown that Clomiphene citrate can have destructive effects on the blood circulation of the ovarian artery and that accompanying with NO in the ovarian blood circulation and endometrial vascular and admitting it can improve pregnancy rate (26).

Another study showed that oral L-arginine supplementation as an NO donor, has improved the ovarian response, endometrial admission, and pregnancy rate in the patients with a very poor ovarian response, who go through assisted reproductive techniques (27).

\section{Material \& Methods:}

\section{Study design:}

In a randomized single blind clinical trial (RCT), 120 women who had polycystic ovarian syndrome (PCOS), were divided into two groups of study and control (60 patients in each group) and were investigated. The subjects were aged between 20 and 35, had one or two open Oviducts in HSG and had been infertile for over three years. The results of the sperm analysis of the subjects' spouses were appropriate for IUI.

\section{Setting and the Duration of the Study:}

The study was carried out in the infertility ward of the Alzahra Teaching Hospital dependent on the Tabriz University of Medical Sciences.

The study took place in 12 months, starting in January, 2012 and ending in March, 2013. During this time, the researchers collected 
raw data, examined patients, and analyzed data.

\section{Ethical Considerations:}

In this study no other extra or unethical intervention was applied for the patients being studied.

All information about the patients is confidential. All of the procedures employed including hysterosonography, sperm analysis, clomiphene administration, and IUI assisted reproductive technique are based on valid references and studies, among the conventional methods of diagnostic and therapeutic that are applied by different physicians for the infertile women.

In this study, there was no ethical prohibition except for the general principles of the application of the medicines and the study is based on existing references. However, before the study, all subjects signed a consent form. It should also be mentioned that the design of this study has been approved by the ethics committee of the Tabriz University of Medical Sciences. Moreover, the study has been registered in IRCT web-site (NO. N1 2013011512146) as a clinical trial study.

\section{Procedures:}

All women (120) who had polycystic ovarian syndrome (PCOS), were aged between 20 and 35, had one or two open Fallopian tubes in HSG, had been infertile for over three years and the results of the sperm analysis of their spouses were appropriate for IUI, were investigated in two groups of study and treatment in 12 months after the study was approved. Sampling of these patients was random and the subjects were divided into two groups employing Rand List software. To determine the body of the sample, the results of a similar study were used. In this study, the ovulation rate was average of $45 \%$ which was statistically significant. Considering $\alpha=0.05$, power of $80 \%$ and the difference of $15 \%$ in the ovulation rate of 180 patients was estimated and 60 patients were included in each group (120 in total). Polycystic ovaries were diagnosed based on (American Society of Reproduction Medicine) ASRM, (European Society of Human Reproductive and Embryo) ESHRE and based on the presence of the two of the following criteria:

1. Oligo-ovulation or an-ovulation

2. Hyperandrogenism (clinical or biochemical)

3. Polycystic ovaries, morphology of PCO is defined base on the presence of 12 or more follicles with the thickness of 2 to $9 \mathrm{~mm}$ in ach ovary or the growth of the ovary body to more than $10 \mathrm{~cm}^{2}$.

The patients in both groups were treated by the daily administration of $50 \mathrm{mg}$ of clomiphene which started on the 3rd -5th day of the menstrual cycle accompanied with exogenous gonadotropins. In the study group, in addition to the above medication, $20 \mathrm{mg}$ nitric oxide tablets (ISMN) produced by Iran medicine company were also administered through the vagina which started on the 3rd -5th day of the menstrual cycle and ended on the diagnosis of ovulation and pregnancy.

Trans-vaginal sonography was administered every other day from day 12 to day 18 of the menstrual cycle to observe mature follicles (equal to or more than $18 \mathrm{~mm}$ ), and diagnose ovulation and the endometrial thickness and were recorded on the last day. If a minimum of one $18 \mathrm{~mm}$ follicle was observed, intramuscular injection of 5000 HCG was administered and IUI was carried out after 34 hours. On the day of IUI vaginal sonography was re-administered to determine ovulation. Pregnancy was determined by the measurement of the $\beta \mathrm{HCH}$ serum in the time of missed menstrual period. Treatment was continued in each group for three cycles. In addition to this, in both groups, age, BMI, FSH rate, LH, Testosterone, and the fasting insulin were also measured.

Inclusion Criteria:

1. Age range of 20 to 35 .

2. One or two open Fallopian tubes in Hysterosonography.

3. Appropriate sperm analysis for IUI;

4. Infertility of over three years in women;

5. Consent to participate in the study.

Variables:

- Patients' age

- Type of infertility

- Type of previous treatment 
- Type of medicine

- Duration of infertility

- Body mass index (BMI)

- Patients' weight

- Patients' height

- Waist

- FSH rate

- LH rate

- Testosterone rate

- Insulin serum rate

- Nitric oxide reception

- Number of mature follicles

- Endometrial thickness

- Presence of ovulation

- Medication side-effect

- $\beta$ HCG results

All above-mentioned information was collected using a pre-designed check list.

Statistical analysis:

Results have been stated in the form of mean \pm standard deviation, frequency and percentage. The statistical software employed is SPSS version 16.

To compare qualitative variables, a student T-test and to compare qualitative variables, a Chi-square test were employed. And when it was required, the Fisher and the McNemar tests were employed in both groups. Every test which had a $\mathrm{P}<.05$ was considered statistically significant.

\section{Results:}

In a randomized single blind clinical trial (RCT), 120 women who had polycystic ovarian syndrome (PCOS), were divided into two groups of study and control (60 patients in each group) and were investigated. The subjects were aged between 20 and 35, had one or two open Fallopian tubes in HSG and had been infertile for over three years. The results of the sperm analysis of the subjects' spouses were appropriate for IUI. Results of this study are as follows.60 women (50\%) were in the study group (group A: patients who were treated with exogenous gonadotropins + clomiphene + nitric oxide (ISMN)) and 60 were in the control group (group B: patients who were treated with exogenous gonadotropins + clomiphene). All participant patients were treated and examined until the end of the study.

Age:

The average age of all of the patients, who were aged between 20 to 35 years, was
$28.3 \pm 4.2$. When groups were considered in isolation, the average age of the women was $28.5 \pm 4.1(22-35)$ in the study group and $28.1 \pm 3.9(21-35)$ in the control group. In this study, the average age difference between the two groups was not statistically significant $(\mathrm{P}=.56)$.

Frequency of the women in different age groups:

Women were divided into three groups based on their age:

In group $A$, there were 13 women in the age group of under 25 (21.7\%), 30 women in the age group of $26-30$ (50\%), and 17 women in the age group of $31-35(28.3 \%)$.

In group B, there were 13 women in the age group of under $25(21.7 \%), 35$ women in the age group of $26-30(58.3 \%)$, and 12 women in the age group of $31-35$ (20\%).

In general, the biggest frequency of the patients in both groups was in the age group of $26-30$. The difference of the frequencies of the two groups of the patients was not significantly significant either $(\mathrm{P}=.53)$.

Description of the Type of the Infertility in the Patients:

The fertility among the patients of the study group was of primary type in 31 cases (51.7\%), and of secondary type in 29 cases (48.3\%).

In the control group, the fertility was of primary type in 36 cases (60\%), and of secondary type in 24 cases (40\%). According to the results of the statistical analysis, the difference of the fertility type between the two groups was not significant $(\mathrm{P}=.23)$.

Description of the Treatments Patients Received before this Study:

In the study group, 26 cases (48.3\%) had already been treated by IUI before the study, while the remaining 31 cases (51.7\%) had not received any IUI before.

In the control group, 35 cases (58.3\%) had already been treated by IUI before the study, while the remaining 25 cases $(41.7 \%)$ had not received any IUI before. The two groups were not significantly different regarding the kind of the treatment they had received before the study $(\mathrm{P}=.18)$.

The kind of medication patients had taken for infertility treatment along with more details and the $\mathrm{P}$ values are illustrated in table 4.1 . 
Description of the duration of the fertility in the patients:

In all patients, the duration of the infertility had been investigated and the one who were infertile for over three years were included in the study. The average duration of the infertility was $4.7 \pm 2.1$ years $(3-8)$ in group $A$, and $5 \pm 2.2$ years $(3-10)$ in group $B$. this difference between the two groups was not statistically significant $(\mathrm{P}=.40)$.

Description of the weight indexes, waist:

The weight indexes of the patients like weight, height, body mass index (BMI), and waist were investigated in this study and compared between the two groups. The details of this investigation along with the $\mathrm{P}$ values are illustrated in table 4-2.

Description of the Para-clinical indexes:

The para-clinical indexes like FSH and $\mathrm{LH}$ rates, Testosterone, and the rate of serum insulin have been measured and compared between the two groups. The details of this investigation along with the $\mathrm{P}$ values are shown in table 4-3.

Description of the Consequences of the Study: The consequences of the study such as the number of the mature follicles, the thickness of the endometrium on the day when HCG was administered, and ovulation were investigated in the women by trans-vaginal sonography on the day of the IUI. The description of these consequences, the comparisons between the two groups along with more details and the $\mathrm{P}$ values are indicated in table 4-4.

\section{Successful Pregnancy Cases:}

At the end of the study, a $\beta$ HCG experiment as the final consequence of the study was carried out in both groups. In the study group with nitric oxide, 10 cases (16.6\%) and in the control group, 8 cases (13.3) had successful pregnancies. The difference of the results of the $\beta$ HCG experiment (successful pregnancy) between the two groups was not statistically significant $(\mathrm{P}=.18)$.

\section{Discussion:}

As it was mentioned, nitric oxide is a free radical gas whose effect does not last long and is made up of L- Arginine and is believed to be the main intervention in the most of the natural functions of the cardiovascular organs, reproductive organs, immune system and digestion (7). Based on the results of several studies, ovaries are able to synthesize nitric oxide and possibly this substance has a role in ovary steroid production, ovulation, and the destruction of the corpus luteum (8).

It seems that nitric oxide does not affect ovaries only when they are under natural conditions. Local effects of nitric oxide in polycystic ovary syndrome and with inexplicable reasons have been have been proved (9).

There are studies that emphasize the effect of nitric oxide on steroid production of ovaries. During animal's sexual cycle, several changes in hormonal activities and in ovaries take place. Nitric oxide activity in the steroid production of the yellow body is important (7). Under the cellular culture conditions, nitric oxide takes the aromatase enzyme activity under control and decreases estradiol production (10).

In this chapter, the aim is to discuss the results of this study and compare them to the findings of the other studies. Therefore, we first point to some of the similar studies in this area.

Elberry et al (2010) in a trial study in Egypt investigated the effects of nitric oxide on the improvement of the successful pregnancy rate in the infertile women with polycystic ovarian syndrome (28).

In the above study which took place in two groups of clomiphene only and clomiphene plus nitric oxide, results indicated that in the study group, because of the follicular maturation, the quality of the cervical mucus and the other endometrial characteristics which resulted from nitric oxide administration, the pregnancy and the ovulation rate also increased (28). In this study, it has been suggested that in the infertile women who suffer from PCOS and are resistant to clomiphene, it is better that prior to the administration of gonadotropin and ovarian laparoscopic surgeries, the ISMN tablets be used in addition to common infertility treatments (28).

In this study also like Elberry et al's, the rate of the successful pregnancy was higher in the study group. That is, in patients who were treated with nitric oxide, 10 cases 
(16.6\%) had successful pregnancies while in the control group, 8 cases $(13.3 \%)$ had successful pregnancies.

However, in contrast to Elberry et al's study, the difference in the successful pregnancy rate between the two groups was not statistically significant $(\mathrm{P}=.18)$.

Also, in contrast to Elberry et al's study, in our study, the patients in both groups were treated with clomiphene and exogenous gonadotropins, and only the study group received the ISMN tablets.

In our study, the endometrial thickness as one of the effective factors in fertility, was $10.3 \pm 2.4 \mathrm{~mm}$ in the study group, and $9.3 \pm 2.4 \mathrm{~mm}$ in the control group. The difference between the two groups was not statistically significant which is in line with the results of Elberry et al's study (28).

Ramsay et al reported the increase in the uterine blood circulation rate and in the Perfusion of the uterine arteries in the women who received nitric oxide. This increase in turn leads to the improvement in the endometrial growth (29).

Results of another similar study in Brazil by Nacul et al (2007) showed that in the patients suffering from PCOS, the level of insulin has a significant negative correlation with NO production (24).

In this study, like the results of Nacul et al's study (24), the rate of the insulin serum in both groups of patients with PCOS was higher than the normal.

In a similar study in Turkey by Turkcuoglu et al (2010), the level of NO in the patients with PCOS was investigated. The results indicated that the level of NO had a significant negative correlation with the level of fasting insulin and also the level of NO is related to the high level of insulin in these patients (25).

Another study showed that oral L-arginine supplementation as an NO donor, has improved the ovarian response, endometrial admission, and pregnancy rate in the patients with a very poor ovarian response, who go through assisted reproductive techniques (27).

Also, in the study by Elberry et al, it was indicated that NO can have Deleterious effects on the quality of embryo and the rate of pregnancy in the cycles of ovarian stimulation. Side-effects of ISMN which is employed through the vagina, have been reported in a few cases and cab be tolerated without any intervention. These side-effects include nausea, feeling the heat, and headache (29).

In our study like Elberry's (29), no sideeffects of ISMN were observed.

In our study, the average duration of the infertility was $4.7 \pm 2.1$ years (3-8) in the study group and $5 \pm 2.2$ years (3-10) in the control group. This difference was not statistically significant between the two groups $(\mathrm{P}=.40)$.

In a review of the similar studies in this area, we could not find any indication that the duration of infertility can affect the response to nitric oxide during infertility (28-30), however, in our study the two groups were not statistically different from each other regarding this factor.

In our study, the average age of all of the participants who were aged between 21 and 35 , was $28.3 \pm 4.2$. When groups were considered in isolation, the average age of the women was $28.5 \pm 4.1(22-35)$ in the study group and $28.1 \pm 3.9(21-35)$ in the control group. It is worth mentioning that this difference was not statistically significant.

Like our study, in Elberry et al's study (29), the difference between the two groups of the study was not statistically significant, however, in contrast to our study; there was a fewer number of patients in Elberry's study. Farzi et al in Gilan University of Medical Sciences investigated the effect of nitroglycerin (NTG) injection prior to embryo transition in assisted reproductive techniques (31). Findings showed that nitroglycerin had no significant effect on the pregnancy rate in the study group compared to the placebo group. This study suggested that more research needs to be done to prove the results (31).As it was mentioned, in our study the two groups were not statistically different regarding the rate of the successful pregnancy $(\mathrm{P}=.18)$. This is in accordance with the results of the study by Frazi (31), but not with the findings of Elberry et al's study(28).It seems that there are other factors like maternal factors or environmental conditions are also at work and can affect the rate of ovulation and 
pregnancy rate. This should be investigated in the future studies.

\section{Conclusion :}

According to the results and because there was not a statistically significant difference between the two groups of the study regarding the rate of successful pregnancy, it can be stated that the employment of nitric oxide tablets in addition to the common treatment should not be more preferable than the employment of the common treatment only in treating the women with polycystic ovarian syndrome. It is suggested that more multi-center studies be carried out including more participants to clarify the effect of nitric oxide in assisted reproductive techniques.

Suggestions for Further Research:

In the current study, it is suggested that:
1.A similar study be carried out on the infertile women with poly cystic ovarian syndrome to investigate the hemostatic status;

2.A similar study be carried out on the infertile women with poly cystic ovarian syndrome to investigate the rate of the changes in homocysteine and fibrinogen and other hemostatic markers in treating infertility.

\section{Conflicts of interest:}

Authors declare that there is no any conflict of interest.

\section{Acknowledgments:}

The authors would like to thanks Department of Gynecology- Alzahra Hospital- Faculty of Medicine, Tabriz University of Medical Sciences.

Figure 4-1. Distribution of the frequencies of the age groups in two groups.

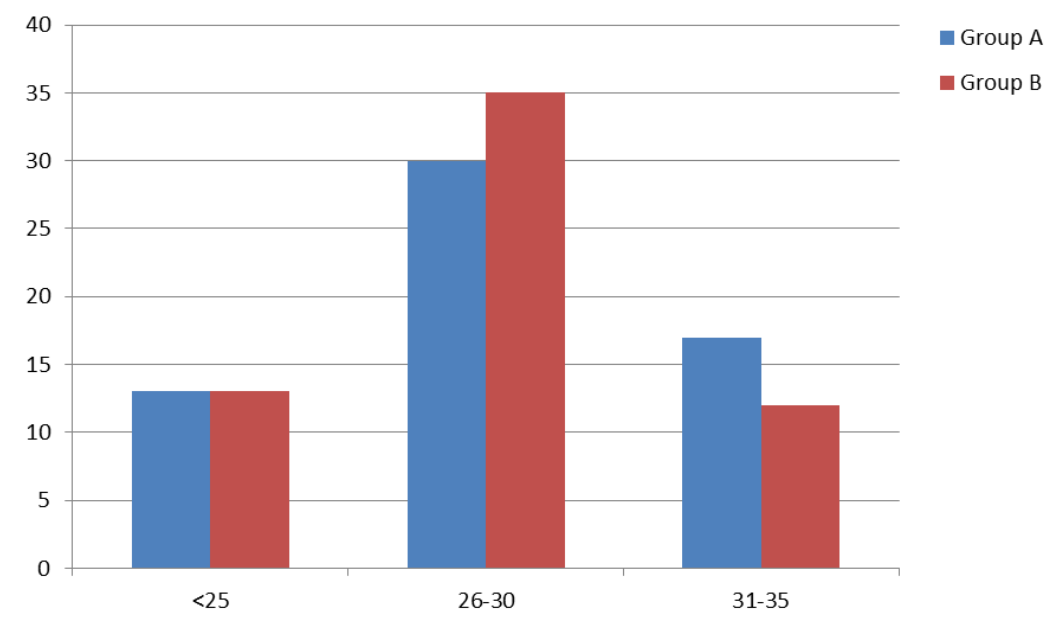

Table 4-1. Comparison of the history of previous medication between the two groups.

\begin{tabular}{|l|c|c|c|}
\hline Medication Type & $\begin{array}{c}\text { Treatment group with } \\
\text { ISMN 60 patients }\end{array}$ & $\begin{array}{c}\text { Control Group } \\
60 \text { patients }\end{array}$ & \multirow{2}{*}{ P value } \\
\hline Clomiphene & $51(\% 85)$ & $55(691 . \%)$ & \multirow{2}{*}{0.003} \\
\hline Clomiphene + NO & $9(\% 15)$ & $5(.4 \% 8)$ & \\
\hline
\end{tabular}


Figure ????? - Comparison of the history of previous medication between the two groups

\section{Comparison of the history of previous medication between the two groups}

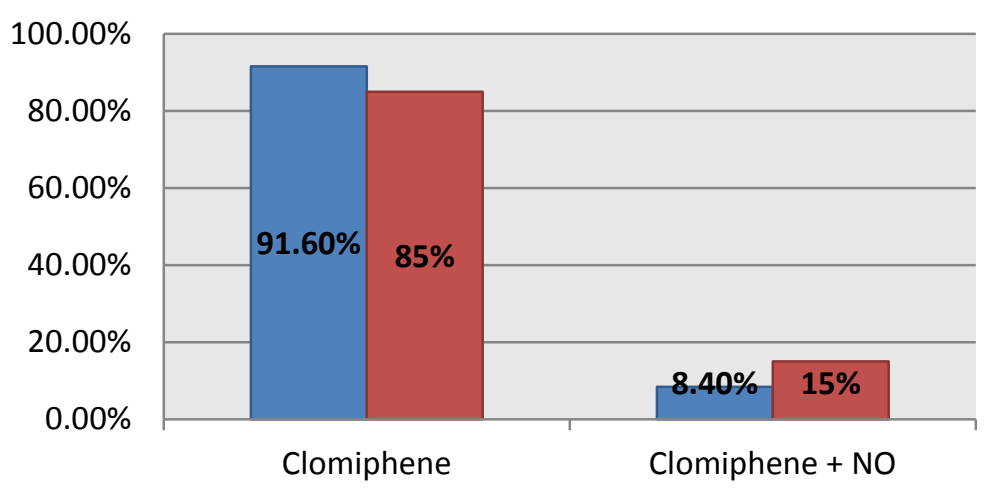

$\square$ Control Group

$\square$ Treatment group with ISMN

Table 4-2. The comparison of the weight indexes and waist between the two groups.

\begin{tabular}{|c|c|c|c|}
\hline Variable & $\begin{array}{c}\text { Treatment group with ISMN } \\
60 \text { patients }\end{array}$ & $\begin{array}{c}\text { Control Group } \\
60 \text { patients }\end{array}$ & P Value \\
\hline Weight $(\mathrm{Kg})$ & $\begin{array}{c}74.8 \pm 10.6 \\
(51-93)\end{array}$ & $\begin{array}{c}71.4 \pm 11.3 \\
(50-89)\end{array}$ & 0.09 \\
\hline Height $(\mathrm{cm})$ & $\begin{array}{c}161.4 \pm 5.6 \\
(148-175)\end{array}$ & $\begin{array}{c}161.1 \pm 7 \\
(140-170)\end{array}$ & 0.83 \\
\hline $\begin{array}{c}28.7 \pm 3.5 \\
\text { Body Mass Index }\end{array}$ & $\begin{array}{c}27.5 \pm 4 \\
(19.5-35.1)\end{array}$ & 0.10 \\
\hline Waist $(\mathrm{cm})$ & $\begin{array}{c}90.9 \pm 9.7 \\
(66-115)\end{array}$ & $\begin{array}{c}90.3 \pm 7.6 \\
(66-107)\end{array}$ & 0.73 \\
\hline
\end{tabular}

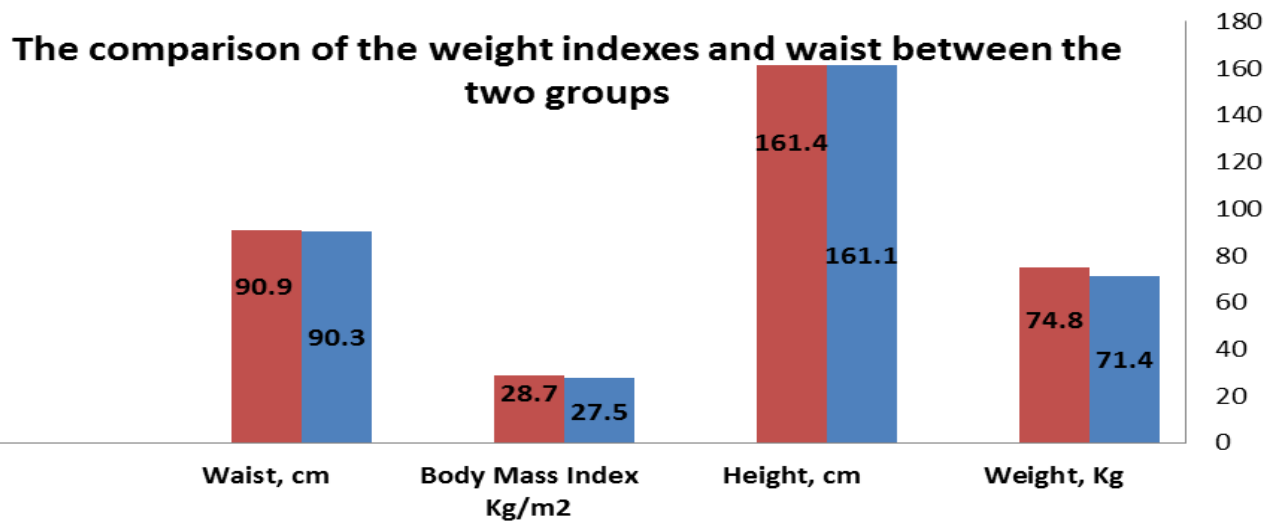

Control Group Treatment group with ISMN 
Table 4-3. The comparison of the para-clinical indexes between the two groups.

\begin{tabular}{|l|c|c|c|}
\hline \multicolumn{1}{|c|}{ Variable } & $\begin{array}{c}\text { Treatment group with ISMN } \\
60 \text { patients }\end{array}$ & $\begin{array}{c}\text { Control Group } \\
60 \text { patients }\end{array}$ & P Value \\
\hline FSH (mIU/L) & $\begin{array}{c}6.48 \pm 3.12 \\
(2.2-14)\end{array}$ & $\begin{array}{c}6.37 \pm 2.79 \\
(2.3-14)\end{array}$ & 0.85 \\
\hline LH (mIU/L) & $\begin{array}{c}8.38 \pm 5.35 \\
(2.1-25)\end{array}$ & $\begin{array}{c}9.99 \pm 5.64 \\
(2.4-16)\end{array}$ & 0.11 \\
\hline Testosterone & $\begin{array}{c}0.64 \pm 0.41 \\
(0.2-2.1)\end{array}$ & $\begin{array}{c}0.58 \pm 0.38 \\
(0.2-2.1)\end{array}$ & 0.41 \\
\hline Ing/mL) & $\begin{array}{c}12.9 \pm 6.1 \\
(5-25.5)\end{array}$ & $\begin{array}{c}12.3 \pm 5.8 \\
(4.4-25)\end{array}$ & 0.63 \\
\hline
\end{tabular}

The comparison of the para-clinical indexes between the two groups

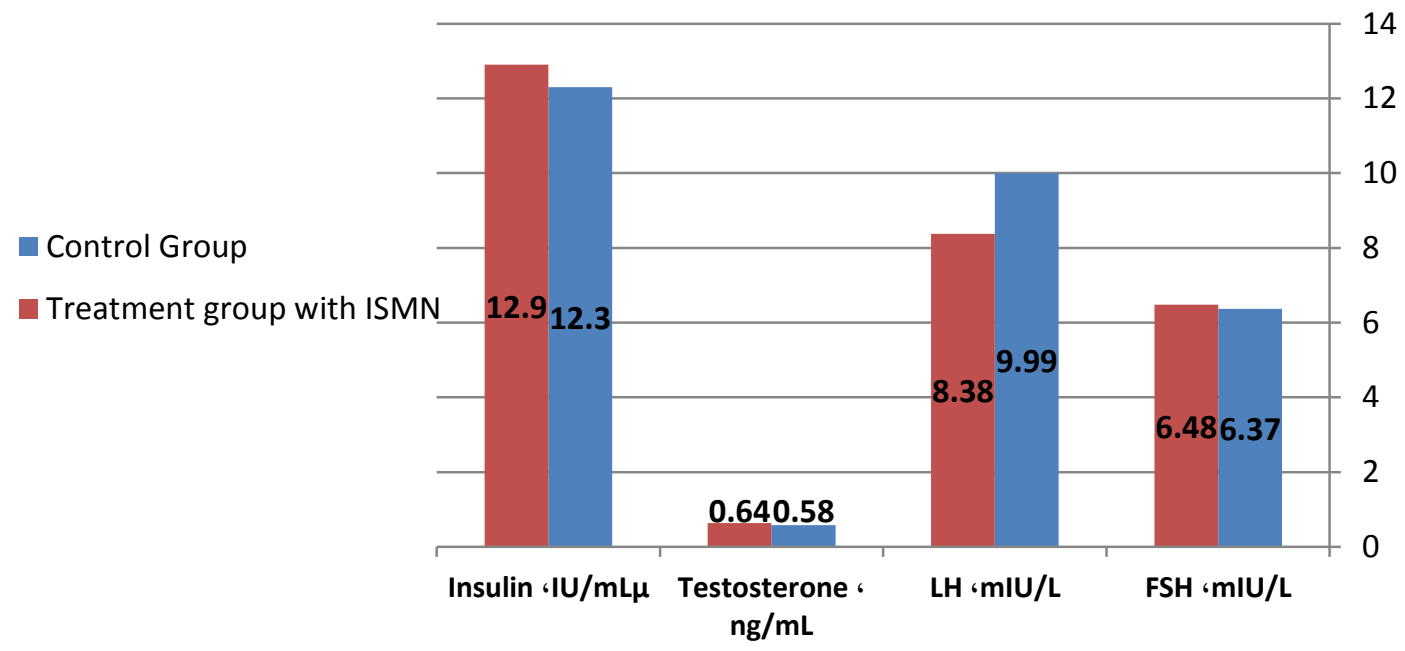

Table 4-4. The comparison of the consequences of the study between the two groups.

\begin{tabular}{|c|c|c|c|}
\hline Variable & $\begin{array}{c}\text { Treatment group } \\
\text { with ISMN 60 } \\
\text { patients }\end{array}$ & $\begin{array}{c}\text { Control Group } \\
\text { 60 patients }\end{array}$ & P Value \\
\hline Endometria & $\begin{array}{c}10.3 \pm 2.4 \\
(4.8-14)\end{array}$ & $\begin{array}{c}9.3 \pm 2.4 \\
(3.3-14)\end{array}$ & 0.02 \\
\hline Thickness, mm & $43(71.7 \%)$ & $52(82.7 \%)$ & 0.035 \\
\hline Presence of ovulation & $\begin{array}{c}3.33 \pm 1.79 \\
(1-6)\end{array}$ & $\begin{array}{c}3 \pm 1.52 \\
(1-6)\end{array}$ & 0.27 \\
\hline Number of the & mature follicles &
\end{tabular}

The comparison of the consequences of the study between the two groups

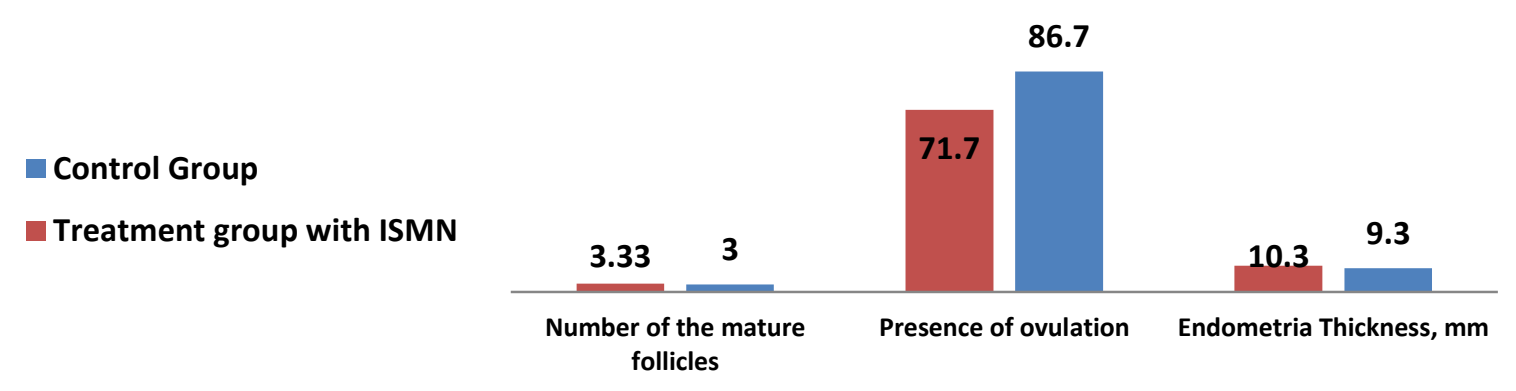




\section{References:}

1. Raval AD, Hunter T, Stuckey B. Statin for women with poly cystic ovary syndrome not actively trying to conceive. Cochrane Syst Rev. 2011; 128:65-85.

2. Sthyapalan T, Smith KA, Coady AM. Atorvastatin therapy decrease androstendione and dehydroepiandrostendione sulphate concentration in patients with polycystic ovary syndrome randomized clinical trial. Ann Clin Biochem. 2012; 49: 80-85.

3. Amin AF, Abd el-Aal DE, Darwish AM, Meki AR. Evaluation of the impact of laparoscopic ovarian drilling on Doppler indices of ovarian stromal blood flow, serum vascular endothelial growth factor and insulin-like growth factor-1 in women with polycystic ovary syndrome. Fertil Steril. 2003; 79(4): 938-941.

4. Edwards RG, Beard HK. Is the success of human IVF more a matter of genetics and evolution than growing blastocysts. Hum Reprod. 1999; 14(1):1-4.

5. Engmann L, Maconochie N, Sladkevicius P, Bekir J, Campbell S, Tan SL. The outcome of in-vitro fertilization treatment in women with sonographic evidence of polycystic ovarian morphology. Hum Reprod. 1999; 14(1):167-171.

6. Fulghesu AM, Angioni S, Frau E. (2007). Ultrasound in polycystic ovary syndrome the measuring of ovarian stroma and relationship with circulating androgens: results of a multicentric study. Hum Reprod. 2007; 22(9):501-2508.

7. Dixit VD, Parvizi N. Nitric oxide and the control of reproduction. AnimReprodSci.2001; 65:1-16.

8. Marinoni E, Iorio R, Villaccio B, Letizia C, Aragona C, Schimberni M, Cosmi EV. Follicular fluid adrenomedullin concentrations in spontaneous and stimulated cycles: relationship to ovarian function and endothelin-1 and nitric oxide. Regul Peptides. 2002; 107:125-128.

9. Cussons AJ, Stuckey BGA, Watts GF. Cardiovascular disease in the polycystic ovary syndrome: New insights and perspectives. Atherosclerosis. 2006; 185:227-239.

10. Weems YS, Lennon E, Uchima T, Raney A, Goto K, Ong A, Zaleski H, Weems CW. Is nitric oxide luteolytic or antiluteolytic. Prostaglandins Other Lipid Mediat. 2005; 8:129-138.

11. Elberry S, Razik M. (2010). Nitric oxide donors increases pregnancy rate in clomiphene citrate treated polycystic ovary infertile patients. Middle East Fertility Society Journal. 2010; 15:106-109.

12. Ramsay B, JohnsonMR,LeoneAM. The effect of exogenous oestrogen on nitric oxide production in women: a placebo controlled crossover study. Br J Obstet Gynecol. 1995; 102:417-419.

13. Nácul AP, Andrade CD, Schwarz P, de Bittencourt PI Jr, Spritzer PM. Nitric oxide and fibrinogen in polycystic ovary syndrome: associations with insulin resistance and obesity. Eur J Obstet Gynecol Reprod Biol. 2007; 133(2):191-196. 
14. Türkçüoğlu I, Engin-Üstün Y, Turan F, Kali Z, Bay Karabulut A, Meydanli M, Kafkasli A. Evaluation of asymmetric dimethylarginine, nitric oxide levels and associated independent variables in obese and lean patients with polycystic ovarian syndrome. Gynecol Endocrinol. 2010; 9:33-42.

15. Honaramooz A, Cook SJ, Beard AP, Bartlewski PM, Rowling NC. (1999). Nitric oxide regulation of gondotropin secretion in prepubertal heifers .J Neuroendocrinol. 1999; 11:667676. 